\title{
AtomicChargeCalculator: interactive web-based calculation of atomic charges in large biomolecular complexes and drug-like molecules
}

\author{
Crina-Maria lonescu ${ }^{1 \dagger}$, David Sehnal ${ }^{1,2,3 \dagger}$, Francesco L. Falginella ${ }^{1}$, Purbaj Pant ${ }^{2}$, Lukáš Pravda ${ }^{1,2}$, \\ Tomáš Bouchal ${ }^{1,2}$, Radka Svobodová Vařeková1,2, Stanislav Geidl ${ }^{1,2}$ and Jaroslav Koča ${ }^{1,2^{*}}$
}

\begin{abstract}
Background: Partial atomic charges are a well-established concept, useful in understanding and modeling the chemical behavior of molecules, from simple compounds, to large biomolecular complexes with many reactive sites.

Results: This paper introduces AtomicChargeCalculator (ACC), a web-based application for the calculation and analysis of atomic charges which respond to changes in molecular conformation and chemical environment. ACC relies on an empirical method to rapidly compute atomic charges with accuracy comparable to quantum mechanical approaches. Due to its efficient implementation, ACC can handle any type of molecular system, regardless of size and chemical complexity, from drug-like molecules to biomacromolecular complexes with hundreds of thousands of atoms. ACC writes out atomic charges into common molecular structure files, and offers interactive facilities for statistical analysis and comparison of the results, in both tabular and graphical form.

Conclusions: Due to high customizability and speed, easy streamlining and the unified platform for calculation and analysis, ACC caters to all fields of life sciences, from drug design to nanocarriers. ACC is freely available via the Internet at http://ncbr.muni.cz/ACC.
\end{abstract}

Keywords: Conformationally dependent atomic charges, Biomacromolecules, Drug-like molecules, Paracetamol, Benzoic acids, Protegrin, Proteasome, Allostery, Chemical reactivity

\section{Background}

Partial atomic charges are real numbers meant to quantify the uneven distribution of electron density in the molecule, and have been used for decades in theoretical and applied chemistry in order to understand the chemical behavior of molecules. Atomic charges are extensively used in many molecular modeling and chemoinformatics applications. With respect to biomacromolecules, charges can elucidate electrostatic effects critical for long range molecular recognition phenomena, protein folding,

\footnotetext{
*Correspondence: jaroslav.koca@ceitec.muni.cz

${ }^{\dagger}$ Crina-Maria Ionescu and David Sehnal contributed equally

${ }^{2}$ National Centre for Biomolecular Research, Faculty of Science, Masaryk

University, Kotlářská 2, 61137 Brno, Czech Republic

Full list of author information is available at the end of the article
}

dynamics and allostery, directed adduction of substrates and egression of products in enzymes, ligand binding and complex formation for proteins and nucleic acids, etc. [1-3]. With respect to drug-like molecules, atomic charges provide information related to reactivity and can be used in the prediction of various pharmacological, toxicological or environmental properties $[4,5]$.

Although, in principle, it is possible to estimate atomic charges based on experimental measurements (e.g., $[6,7])$, such calculations are impractical. Most commonly, atomic charges are estimated based on theoretical approaches. Quantum mechanical (QM) approaches first solve the Schrödinger equation [8] and calculate the electron density using a combination of theory level and basis set. They then partition the obtained molecular

\section{Chemistry Central}


electron density (or a density-derived quantity) into atomic contributions (atomic partial charges) according to various population analyses [9-19]. Empirical approaches to atomic charge calculation (e.g., [20-27]) have been proposed as resource-efficient alternatives to QM approaches, as they do not require the demanding step of solving the Schrödinger equation. In particular, approaches based on the equalization of molecular electronegativity [22, 23, 28-35] are of interest because they are sensitive to both the chemical environment and molecular conformation.

Due to the essential role of atomic charges, many modeling tools currently include atomic charge calculation capabilities (e.g., [36-50]). However, in the case of druglike molecules, only a few tools can provide QM quality charges which respond to changes in conformation or chemical environment without needing to first obtain the QM electron density or electrostatic potential [47, $48,50]$. Moreover, these tools are not sufficiently general, resource-efficient or interactive. In the case of biomacromolecules, no freely available software tool can readily provide atomic charges of QM quality, despite repeated reports that such quality is necessary [51-54]. We have accepted these challenges and set out to provide a robust and accessible software solution for atomic charge calculation for molecules of all nature and size.

This contribution presents the AtomicChargeCalculator (ACC), a free web application for the calculation and analysis of atomic charges which respond to changes in molecular conformation and chemical environment. The calculation is based on the electronegativity equalization method (EEM [22]), a powerful empirical approach which can provide atomic charges similar to those generated by various $\mathrm{QM}$ approaches, but using much lower computational resources. Along with the classical EEM algorithm, ACC implements two additional EEM approximations with increased efficiency, specifically tailored for studying very large molecular systems. A single calculation may take from less than a second (small molecules), to a few minutes (large biomacromolecular complexes). ACC outputs the most common molecular structure formats containing atomic charges. Additionally, it provides facilities for statistical analysis and comparison of the results, in tabular and graphical form. ACC also includes interactive 3D visualization of the molecules based on atomic charges. A command line version is also available.

\section{Implementation}

The challenge was to provide a robust web based software solution for atomic charge calculation for molecules of all nature and size. Therefore, we first focused on identifying and optimizing a suitable algorithm for atomic charge calculation, and then on implementing the optimal workflow for setting up an ACC calculation and interpreting the results.

The application was constructed using the client-server architecture (Fig. 1): the charge computation is carried out on the server and implemented in the C\# programming language. The JavaScript Object Notation (JSON) is used to transfer data to the client that provides the user interface (UI) implemented using HTML5 and JavaScript. Additionally, the UI uses the WebGL technology to provide a custom built $3 \mathrm{D}$ visualization of the computed charges.

\section{Computational details}

The Electronegativity Equalization Method (EEM) is the general approach followed by ACC to calculate atomic charges. EEM-based methods have been successfully applied to zeolites and metal-organic frameworks, small organic molecules, polypeptides and proteins [55-61].

EEM is an empirical approach which relies on parameters usually fitted to data from reference QM calculations. The values of atomic charges computed using EEM support chemical reasoning, and generally correlate well with values from reference QM calculations. The accuracy of each set of EEM parameters is documented in the respective literature. On the other hand, classical EEM approaches incorrectly predict superlinear scaling of the polarizability with increasing molecular size, making the models developed on small molecules difficult to transfer to extended systems like biomacromolecules $[62,63]$. This artifact can be

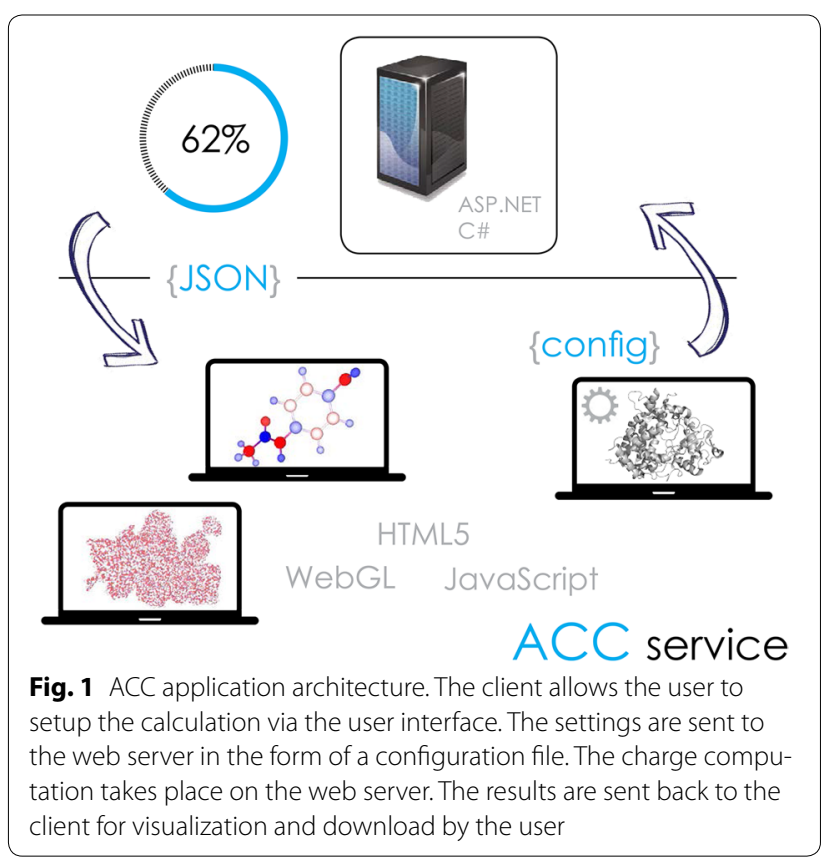


tempered by applying charge conservation constraints to small molecular units. Such extensions to EEM have been proposed [24, 64].

Computationally efficient implementations of EEMbased methods are integrated in tools specialized for reactive molecular dynamics simulations [65] and for generating conformers of drug-like molecules [50, 66]. However, ACC is the first to implement EEM in a manner which is not only computationally efficient, but also independent of the subsequent intended application, and specifically designed to allow users with little background in computational sciences to run charge calculations and interactively analyze the results.

ACC can solve the EEM matrix equation (see the Computational details section of the Additional file 1) if the following input is provided: the 3D structure of the molecular system, the total molecular charge, and a set of EEM parameters. Solving the EEM matrix equation requires solving a dense system of equations. The computational complexity of this procedure is $\mathcal{O}\left(N^{3}\right)$. The space complexity, which refers to the memory required to store the EEM matrix, is $\mathcal{O}\left(N^{2}\right)$, where $N$ is the number of atoms. For very large molecules with tens of thousands of atoms, the EEM approach is too demanding on conventional desktop hardware. We thus propose two new approaches for solving the EEM matrix, namely EEM Cutoff and EEM Cover. These approaches work by splitting the EEM matrix into multiple smaller matrices.

Within the EEM Cutoff approach, for each atom in the molecule, ACC generates a fragment made up of all atoms within a cutoff radius $R$ of the original atom. Thus, for a molecule containing $N$ atoms, the EEM Cutoff approach solves $N$ smaller EEM matrices, for a set of $N$ overlapping fragments of the original molecule. EEM Cutoff effectively reduces the time complexity of the calculation to $\mathcal{O}\left(R^{6} N+R^{2} N \log N\right)$, and the space complexity to $\mathcal{O}\left(R^{4} N+N \log N\right)$. A detailed description of the EEM Cutoff approach is given in the Computational details section of the Additional file 1 .

To further enhance the run-time and memory efficiency of calculations in ACC, we propose EEM Cover, an approach for tackling molecules with hundreds of thousands of atoms. EEM Cover also splits the EEM matrix into smaller matrices, but it generates fragments only for a subset of atoms in the molecule. While the asymptotic complexity remains the same, the number of EEM matrices that need to be solved is reduced by at least 50 \% compared to EEM Cutoff, while maintaining high accuracy. A detailed description of the EEM Cover approach is given in the Computational details section of the Additional file 1.

\section{Workflow}

The ACC workflow is organized into four phases, namely: upload, setup, calculation and results. Each phase is characterized by a set of operations as follows:

1. Upload molecules Multiple molecules can be uploaded in the most common file formats (PDB, $\mathrm{PDBx} / \mathrm{mmCIF}$, PQR, MOL, MOL2, SDF, or .zip with multiple files of a suitable format). The molecular structures should be complete and properly protonated. There is no limitation regarding the size, number or nature of the chemical entities in a single structure file (proteins, nucleic acids, ligands, water, etc.), as all these are loaded and identified as a single molecule within ACC. The total size of the upload is limited to $50 \mathrm{MB}$.

2. Setup Upon uploading the molecule(s), ACC parses the molecular structure to identify the number and types of atoms in the system, as well as the interatomic distances. Based on this information, ACC tries to prefill the submission form with suitable default settings (see the Default settings section of the Additional file 1). These settings can be adjusted by the user before the calculation is started. Each distinct setup (Fig. 2) will result in a certain number of ACC

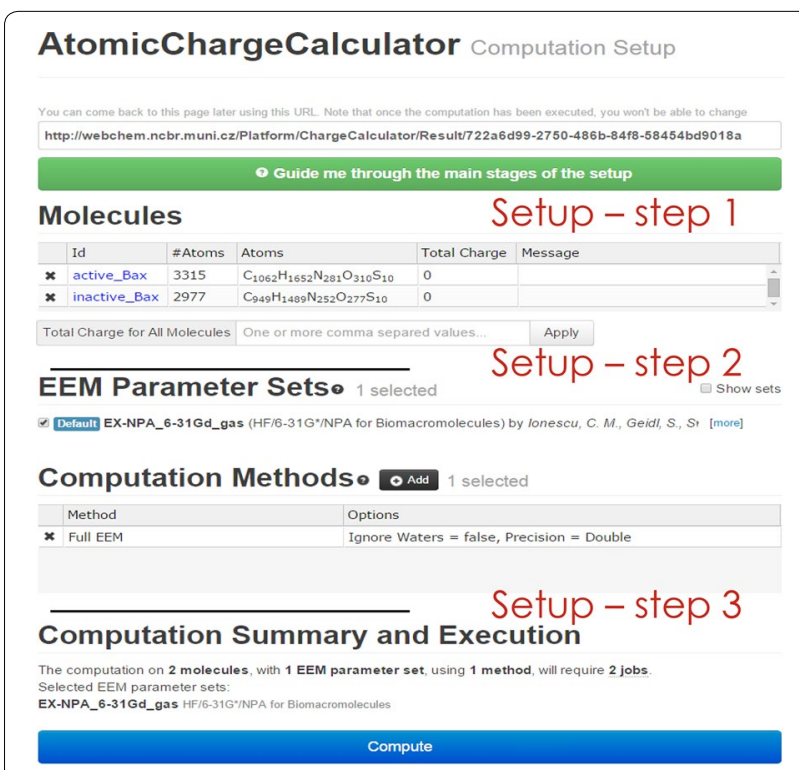

Fig. 2 Setup of jobs in AtomicChargeCalculator. The setup of an ACC calculation takes place in three steps, each step referring to one of three aspects: the molecule and its total charge, the set of EEM parameters to be used in the EEM equation, and the computation options. These three aspects uniquely define an ACC job. A single setup may lead to running several ACC jobs. Based on the information in the uploaded structure files, ACC suggests a default setup, which can be adjusted by the user prior to starting the calculation. Explanations are available in the interactive guides, tool tips and Wiki pages 
jobs, each defined by the molecule, total molecular charge, the set of EEM parameters, and the computation options. For the command line version of ACC, the setup workflow is identical to the steps described below, and is scripted into a configuration file.

2.1. Total molecular charge The total molecular charge quantifies the amount of charge that will be distributed across the molecule during the EEM calculation. By default, ACC assumes that all uploaded molecules are neutral. The user must provide the correct total molecular charge for each non-neutral molecule uploaded.

2.2. Set of EEM parameters EEM employs special parameters for each type of atom $(\mathrm{H}, \mathrm{C}, \mathrm{N}, \mathrm{O}$, halogens, metals, etc., depending on the target molecules). EEM parameters are generally developed based on reference QM calculations. The applicability domain of a given EEM parameter set is generally limited to the target molecules, and closely related to the applicability domain of the particular QM approach used as reference. Performance is further influenced by the procedure used when fitting the EEM parameters to the reference data. Many EEM parameter sets have been published in literature, and are available in ACC as built-in sets [28, 34, 67-70] with full information regarding the parameter development procedure (atom types covered, target molecules, QM reference data, literature reference). By default, ACC tries to select one of these sets based on the atom types present in the uploaded molecules. The user can select a different set of EEM parameters by choosing from the list of available built-in sets, or even uploading customized sets in an XML template. Multiple sets of EEM parameters can be tested in a single ACC run.

2.3 Computation options ACC may compute atomic charges based on one of the three available EEM approaches implemented, namely Full EEM, EEM Cutoff, and EEM Cover. Further options refer to the precision (64 or 32-bit representation of numbers), cutoff radius parameter, and including water molecules into the calculation. By default, ACC picks computation options most suitable to the size of the uploaded molecules. These computation options can be adjusted by the user. Up to 10 computation options can be tested in a single ACC run.

3. Calculation Once the setup phase is complete, the calculation is launched. A single ACC run may con- sist of multiple atomic charge calculation jobs. Each job is uniquely defined by the molecule, total molecular charge, set of EEM parameters, and computation options, and produces one set of atomic charges. Each job may use a different amount of time and memory resources, depending on the size of the molecule and the complexity of the computation.

4. Results The ACC results are organized into hierarchical reports which are stored on the server for download or inspection for up to a month, at a unique URL visible only to the user. The command line version of ACC produces the same overall and single molecule reports described below, but does not facilitate interactive $3 \mathrm{D}$ visualization.

4.1. The overall report contains information and downloadable content (molecular structure files containing atomic charges, statistics of the results, information about all jobs) for all molecules. Single molecule reports are also accessible from here.

4.2. The single molecule report (Fig. 3), which can be downloaded or examined directly in the browser, consists of a few sections:

4.2.1. Summary report containing general information about the input molecule (molecular formula, total charge), calculation setup, a list of all sets of charges produced during the calculation, information about all ACC jobs (duration, warnings, errors) for that molecule.

4.2.2. Interactive list of values for all sets of charges produced by all ACC jobs for that molecule. The atomic charges and residue charges are given.

4.2.3. Statistics within each set of charges, in both tabular and graphical form. The statistics are available for both atomic and residue charges, and are computed for relevant properties such as chemical element, type of residue, etc. The statistical indicators are the minimum value, maximum value, standard deviation, average, median, etc.

4.2.4. Pairwise comparison statistics between sets of charges resulted from different ACC jobs, or uploaded by the user. A graphical representation for each comparison is also provided. The comparison is available for atomic and residue charges. The comparison indicators computed are the squared Pearson's correlation coefficient, Spearman's rank correlation coefficient, RMSD, sum of absolute differences.

4.2.5. Interactive $3 \mathrm{D}$ visualization of molecules. The 3D model can be built based on atomic positions, 


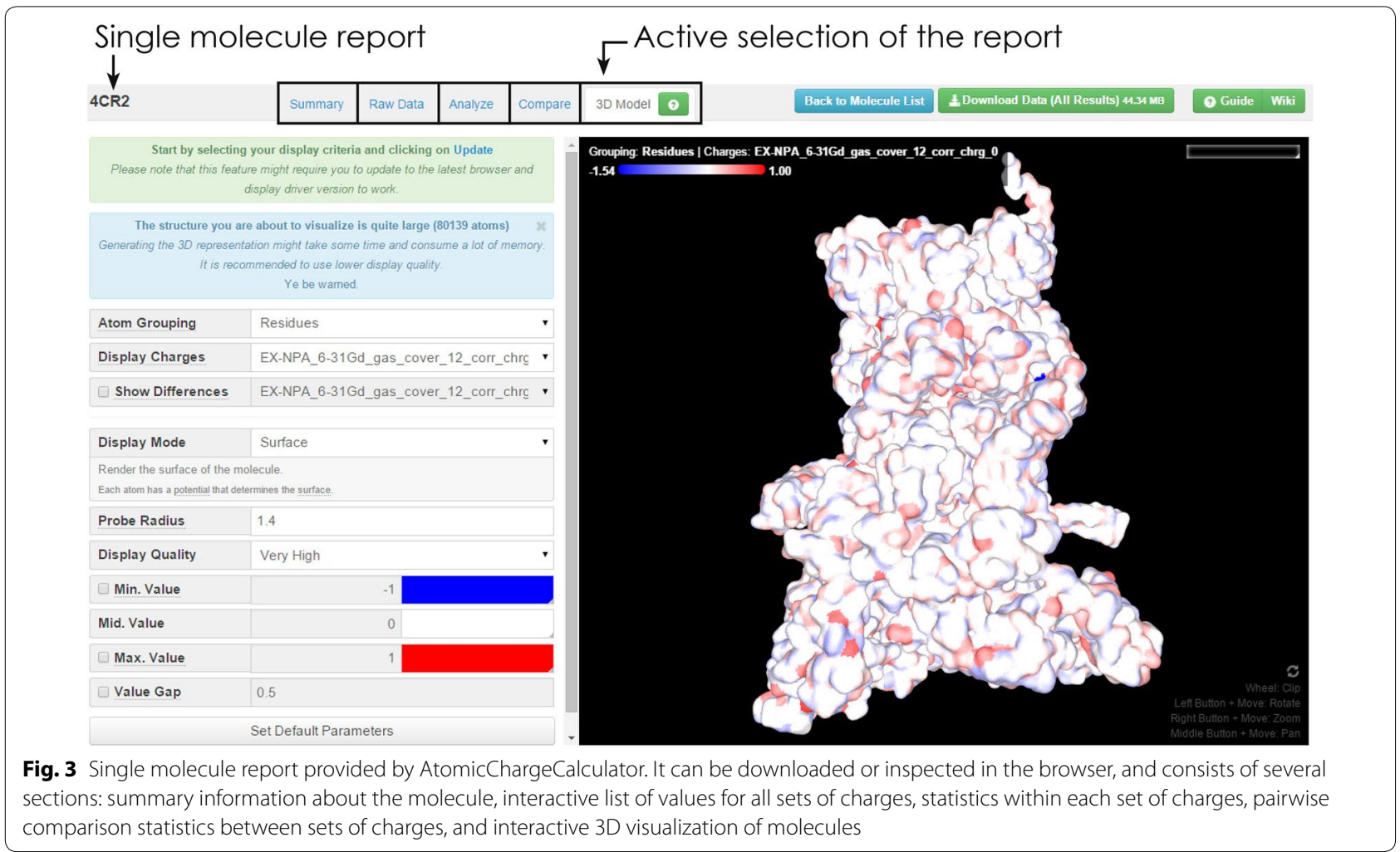

and colored based on atomic charges, or built based on residue positions, and colored based on residue charges. The coloring scheme can also use differences in charges resulted from distinct ACC jobs, or uploaded by the user.

The applicability of ACC is limited by three main aspects: related to the concept of atomic partial charges and its definitions, related to the concept of EEM and its parameters, and related to the $3 \mathrm{D}$ structure of the molecule and its total charge. These aspects are discussed in detail in the Limitations section of the Additional file 1 .

Full documentation explaining the methodology, functionality and interface, along with interesting examples are provided on the web page. Embedded interactive guides assist first-timers and beginners in setting up their calculations and interpreting the results. A command line version of the application is available as an executable for users who wish to streamline more complex calculations.

\section{Results and discussion} Implementation benchmark

We have evaluated the accuracy and computational efficiency of the EEM Cutoff and EEM Cover approaches in a benchmark. The evaluation was performed against reference calculations which solved the full EEM matrix, with a few exceptions. We give here a brief overview (Fig. 4), whereas the full details can be found in the Benchmark section of the Additional file 1. Both EEM Cutoff and EEM Cover are sufficiently accurate, but EEM Cutoff is slightly more accurate. Using a cutoff radius of $8 \AA$ may lead to deviations of up to $0.015 e$, but on average less than $0.008 e$. Using a cutoff radius of $12 \AA$ may lead to deviations of up to $0.008 e$, but on average less than $0.004 e$. The approaches are time efficient compared to Full EEM only when the molecule contains at least 10,000 atoms, but they are always more memory efficient.

Below we provide a few brief examples of uses for AtomicChargeCalculator in the form of case studies. These case studies are focused on a direct interpretation of the ACC results, and show how important hints about the reactivity of a molecule can be obtained in just a few seconds.

\section{Case study I: atomic charges and chemical reactivity in small drug-like molecules}

$N$-acetyl-p-aminophenol, commonly known as paracetamol, is a widely used analgesic and antipyretic. Its mechanism of action is believed to be the inhibition of the protein cyclooxygenase 2 , regulating the production of 

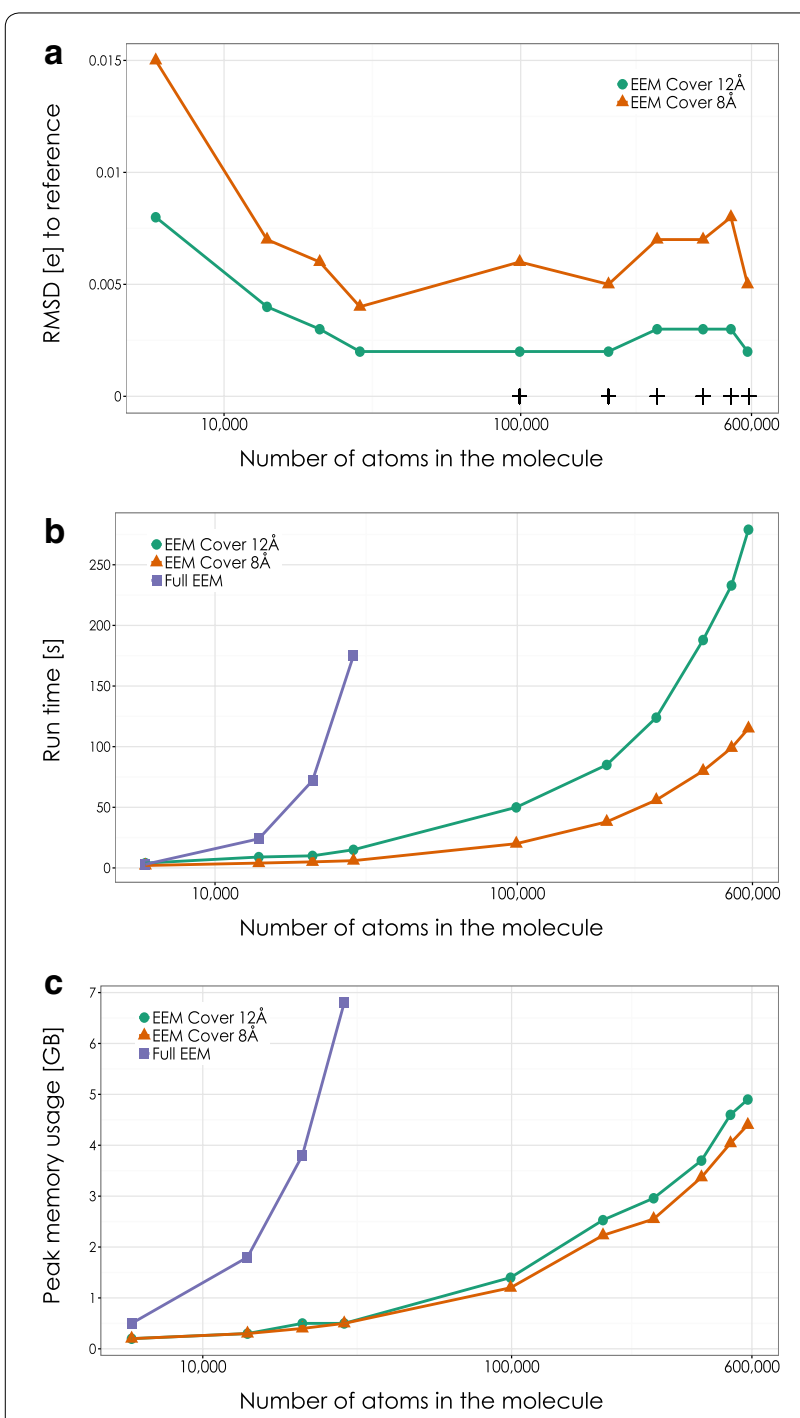

Fig. 4 Benchmark of the EEM Cover approach. Full details can be found in the Benchmark section of the Additional file 1. a EEM Cover is sufficiently accurate, and its accuracy increases with the value of the cutoff radius. The root mean square deviation (RMSD) is calculated by comparison against the reference calculation which solves the entire EEM matrix (Full EEM), using 64-bit precision numbers. Due to the limitation on computational resources, for some molecules the reference calculation was *EEM Cutoff, with a cutoff radius of $17 \AA$ A. b EEM Cover is significantly faster than Full EEM only for molecules with over 10,000 atoms. c EEM Cover is always more memory efficient than Full EEM

pro-inflammatory compounds [71]. The metabolic breakdown of paracetamol has been the subject of intense study, since it holds the key to both its therapeutic action and toxicity.

We calculated atomic charges in paracetamol using ACC. The geometry of the paracetamol molecule corresponded to the ideal coordinates [wwPDB CCD: TYL]. The default ACC settings were used. The computation took less than $1 \mathrm{~s}$, and the complete results are available on the ACC web page at http://ncbr.muni.cz/ACC/ CaseStudy/Paracetamol.

A quick analysis reveals that the phenolic $\mathrm{H}$ (position HO4 in Fig. 5) is the most acidic proton (highest positive charge) in the molecule, suggesting a faster and easier dissociation of this $\mathrm{O}-\mathrm{H}$ bond. Indeed up to $90 \%$ of metabolic degradation happens at position $\mathrm{HO} 4$ (glucuronidation, sulphonation) [72]. Additionally, up to $15 \%$ of metabolic degradation involves oxidation at the phenolic (HO4) and amidic positions ( $\mathrm{HN}$ ) [72], the two most positive $\mathrm{H}$ in the paracetamol molecule. While paracetamol is a very small molecule with few polar sites, the same principle can be applied in reasoning out highly reactive sites in more complex molecules.

Having found out that the most probable dissociation site on the paracetamol molecule is the phenolic $\mathrm{H}$, we were able then to calculate the acid dissociation constant $\mathrm{p} K_{a}$, a property which significantly affects the ability of the drug to cross cellular membranes and thus exert its therapeutic effect. For this purpose we used Quantitative Structure-Property Relationship (QSPR) modeling, as atomic partial charges have been shown to be successful QSPR descriptors in $\mathrm{p} K_{a}$ prediction [58, 73, 74], and QSPR models are available in literature for this purpose. Because the dissociating group on paracetamol is phenolic, we chose a QSPR model specifically developed for the prediction of $\mathrm{p} K_{a}$ in phenols [58], and which employed descriptors based on the EEM charges we computed in our interpretation of local reactivity in paracetamol. The necessary descriptors consisted of the partial charges on the phenolic oxygen (qO4) and hydrogen $\left(\mathrm{q}_{\mathrm{HO}}\right)$, and on the carbon atom binding the phenolic group $\left(\mathrm{q}_{C 4}\right)$. The equation and parameters of the QSPR model are given in Fig. 5.

We thus computed a $\mathrm{p} K_{a}$ value for paracetamol of 9.36, which is close to the experimental value of 9.38 [75]. The computed $\mathrm{p} K_{a}$ suggests that paracetamol is completely unionized at stomach $\mathrm{pH}$, and only $1.1 \%$ ionized at physiological $\mathrm{pH}$, therefore highly efficient at crossing cellular membranes both via oral and intravenous delivery.

While the above described approach was able to provide useful information for paracetamol, it is important to keep in mind that there are limitations to the accuracy of EEM charges. We illustrate such limitations on a series of benzoic acid derivatives. For this purpose, we downloaded the structures of 45 molecules representing benzoic acid derivatives from the NCI Open Database [76]. The data set contained benzoic acids with a wide range of donating and accepting substituents on the phenyl ring in $\mathrm{o}^{-}, \mathrm{m}-$ and $\mathrm{p}-$ positions (Fig. 6a; Additional file 1: Table S3). We chose only compounds for which $p K_{a}$ values were available in Physprop [77]. Furthermore, we did 


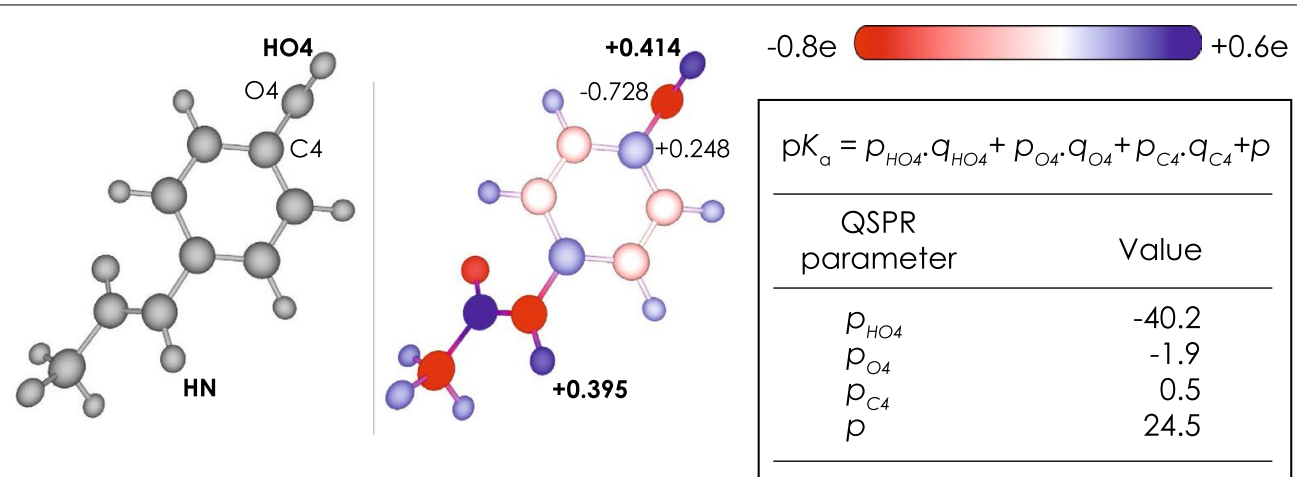

Fig. 5 Atomic charges reveal reactive sites involved in the metabolic degradation of paracetamol. In the picture on the right, the atoms are colored according to their charge. The majority of metabolic degradation of paracetamol (glucuronidation, sulphonation, oxygenation) involves the phenolic (HO4) and amidic ( $\mathrm{HN}$ ) positions, the two most acidic protons in the paracetamol molecule (labels marked in bold) [72]. The relatively higher positive charge on these $\mathrm{H}$ atoms marks more active bonds compared to the rest of the molecule. In the equation of the QSPR model, qHO4 is the charge on the phenolic $\mathrm{H}, \mathrm{q}_{O 4}$ is the charge on the phenolic $\mathrm{O}, \mathrm{q}_{\mathrm{C} 4}$ is the charge on the $\mathrm{C}$ binding the phenolic $\mathrm{OH}_{\text {, and }} \mathrm{p}_{H O 4}$, $\mathrm{p}_{\mathrm{O}}$, $\mathrm{P}_{\mathrm{C} 4}$ and $\mathrm{p}$ are the parameters of the QSPR model, taken from the QSPR model 3d EEM Ouy2009_elem [58]

not include compounds with halogens because the EEM parameter set used in this particular case study cannot treat halogens.

We first wanted to know if and how the effect of different substituents in different positions on the phenyl ring is visible on the charge of the atoms of the carboxyl group. For this purpose, we used Gaussian [36] to compute reference QM atomic charges from a natural population analysis on the electron density obtained at the B3LYP/6-31G* level of theory. Despite the different nature and position of the substituents, the spread of reference QM charges for the atoms in the carboxyl group is very narrow (Fig. 6b). Specifically, the QM values for $\mathrm{O} 1$ are within $0.06 e$, the values for $\mathrm{O} 2$ within $0.01 e$, and the values for $\mathrm{H}$ within $0.01 e$. On the other hand, the EEM parameter set employed here is expected to reproduce the reference QM values within $0.09 e$ [34]. Based on the documented accuracy, we do not expect EEM charges to reflect suitable changes based on the nature or position of the substituents. We used ACC to compute EEM charges for the benzoic acid derivatives, in the same manner as for paracetamol. The computation took less than $2 \mathrm{~s}$, and the complete results (structures, QM charges, EEM charges) are available on the ACC web page at http://ncbr.muni.cz/ACC/CaseStudy/ BenzoicAcids.

Indeed, we found that EEM charges could not accurately reflect the QM spread for the charges on the carboxyl atoms (Fig. 6b). We then wondered if this accuracy, though unable to reflect suitable changes depending on the nature or position of the substituents, was sufficient to build acceptable QSPR models for $p K_{a}$ prediction. No suitable QSPR models are available for benzoic acids, but such models for aliphatic carboxylic acids have been reported $[58,78]$. The descriptors used by these QSPR models consist of charges on the atoms of the carboxylic group in both the neutral $\left(q_{H}, q_{O 1}, q_{O 2}, q_{C 1}\right)$ and dissociated forms $\left(q_{O 1 D}, q_{O 2 D}, q_{C 1 D}\right)$. We thus built QSPR models for benzoic acids based on these descriptors (Fig. 6c). We obtained the structures of the dissociated acids by removing the carboxylic $\mathrm{H}$ atoms. We computed EEM charges for the dissociated molecules using the same ACC setup, but setting the total charge for each molecule at -1 . We then built QSPR models using multilinear regression. We performed a 5-fold cross-validation of the QSPR models, whereby, in each round, 35 randomly chosen molecules were used to train the model, and the remaining 10 molecules were used to validate the model. The QSPR model parameters and full details of the crossvalidation procedure are given in the Additional file 1: Table S4. The models showed adequate predictive capability (Fig. 6c; Additional file 1: Table S4). On average, the mean absolute error during validation was $0.27 p K_{a}$ units compared to experiment, suggesting that EEM charges can be used to predict dissociation constants for benzoic acids, despite their inability to reflect local changes caused by different substituents.

\section{Case study II: atomic charges and activity of antimicrobial peptides}

Protegrins are a family of antimicrobial peptides active against a wide range of pathogens [79]. Protegrin-1 (PG1, Fig. 7) has been intensely studied for its potential in treating infections caused by antibiotic resistant bacteria [80-82]. PG1 shows activity against several pathogens, but also toxicity against the host. Useful mutations are those which maintain the antimicrobial activity, and at the same time reduce toxicity [83]. 


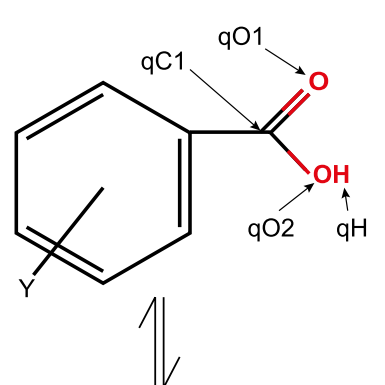

qO1D

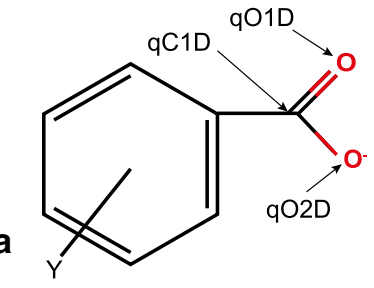

$\mathrm{p} K_{a}=p_{H} \cdot q_{H}+p_{O 1} \cdot q_{O 1}+p_{O 2} \cdot q_{O 2}+p_{C 1} \cdot q_{C 1}+$

$+p_{O 1 D} \cdot q_{O 1 D}+p_{O 2 D} \cdot q_{O 2 D}+p_{C 1 D} \cdot q_{C 1 D}+p$

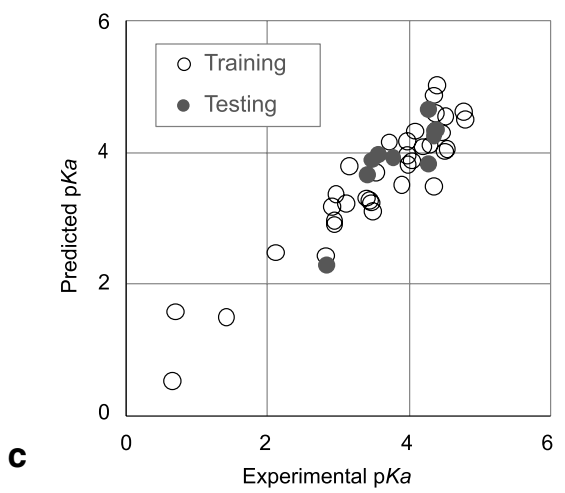

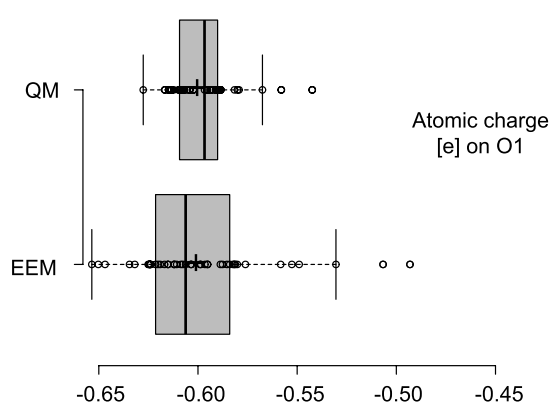

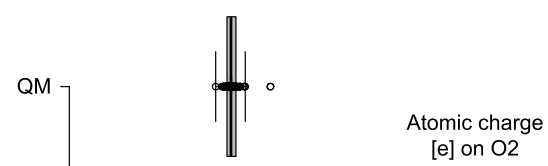

[e] on $\mathrm{O} 2$
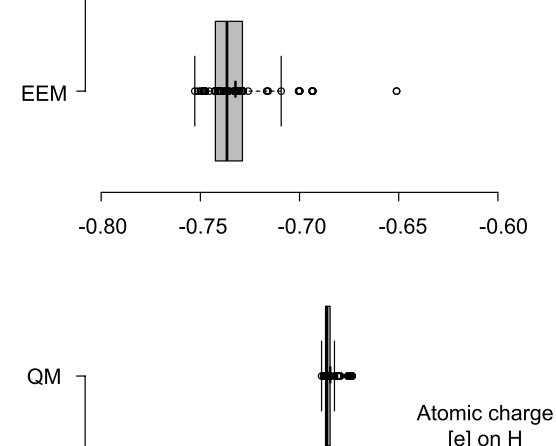

[e] on $\mathrm{H}$

b

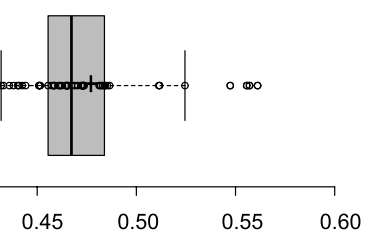

Fig. 6 Limitations of EEM charges illustrated on a series of substituted benzoic acid derivatives. a Denotation of relevant atomic charges in the neutral and dissociated molecules. $\mathbf{b}$ The reference QM charges have a narrow spread despite the different position and wide range of electron donating or withdrawing effects of the subsituents. EEM charges are not accurate enough to reflect the small changes induced by different substituents. c The QSPR descriptors are EEM charges of the atoms of the carboxylic group in both the neutral $\left(q_{H}, 9_{01}, 9_{02}, 9_{C}\right)$ and dissociated forms (9O1D, $902 \mathrm{D}$ , $\left.q_{C 1 D}\right)$. The symbols $p_{H}, p_{O}, p_{O 2}, p_{C 1}, p_{O 1 D}, p_{O 2 D}, p_{C 1 D}$ and $p$ are parameters of the QSPR model. The graph displays the correlation between experimental $p K_{a}$ values, and the values predicted by one of the QSPR models developed in this study. EEM charge descriptors are sufficiently accurate for the prediction of dissociation constants of benzoic acid derivatives

We calculated atomic charges in PG1 using ACC. The geometry of the PG1 molecule corresponded to a low energy NMR model [PDB: 1PG1] [84]. The system contained all $\mathrm{H}$ atoms expected at $\mathrm{pH} 6.5$, as they were listed in the NMR model. The total molecular charge was +7 , owing to the many ARG residues. The EEM parameter set used was EX-NPA_6-31Gd_gas [70], but it was necessary to add to this set EEM parameters for deuterium (D), because this element was present in the input file. The EEM parameters for $\mathrm{D}$ were identical to the parameters for $\mathrm{H}$. The rest of the ACC default settings were kept. The computation took less than $1 \mathrm{~s}$, and the complete results are available on the ACC web page at http://ncbr.muni. $\mathrm{cz} / \mathrm{ACC} / \mathrm{CaseStudy} /$ Protegrin.

The calculation produced one set of atomic charges. PG1 contains 18 residues, and rather than analyzing atomic charges, we analyzed the residue charges, which are also reported by ACC (Fig. 7b). PG1 is special because of its high positive charge. It contains 6 ARG residues. However, not all have the same charge. In particular, ARG at positions 4 and 9 have the least positive charge (around $+0.5 e$ ), whereas the rest have much higher positive charge (over $+0.8 e$ ). Keeping in mind that these charges are likely affected by the polarizability 


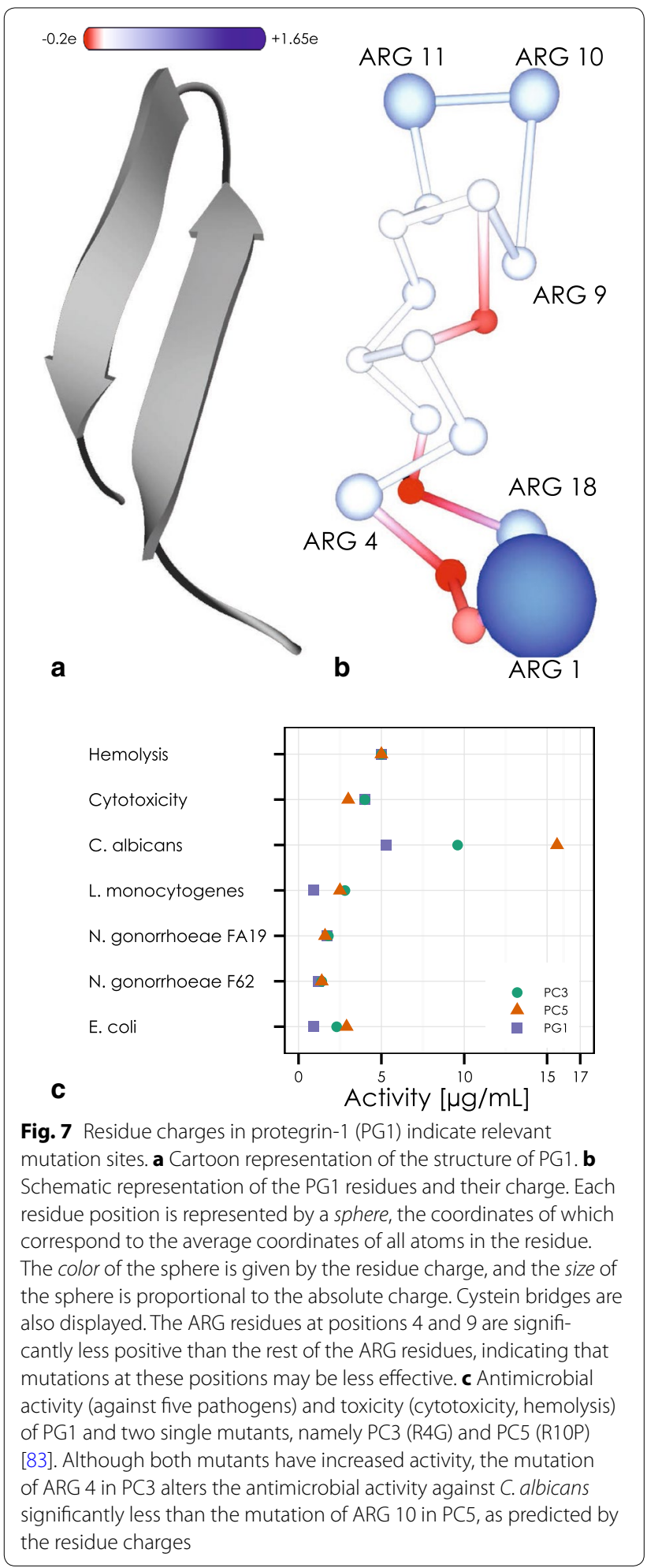

exaggeration artifact of EEM described in the Computational details section, the results suggest that mutations of ARG into a neutral residue at positions 4 or 9 would have a lower effect than mutations at positions $1,10,11$ or 18.

A literature search reveals that many mutants and derivatives of PG1 have been studied [83, 85-87]. In particular, Ostberg and Kastnessis [83] have logged the antimicrobial and toxic activity of sixty-two PG1 mutants and PG1-analogue peptides. Out of these, there are two single point mutants where one ARG was mutated into a neutral residue. These mutants are PC3 (R4G) and PC5 (R10P). The study found that, indeed, the mutation of ARG 4 in PC3 alters the antimicrobial activity against $C$. albicans significantly less than the mutation of ARG 10 of PC5 (Fig. 7c). Such biologically relevant insight can be gained by analyzing the residue charges on a single structure of PG1.

\section{Case study III: atomic charges and allostery of large biomacromolecular complexes}

The $26 \mathrm{~S}$ proteasome is a large biomacromolecular complex which facilitates the targeted degradation of intracellular proteins, and thus plays an essential role in keeping protein homeostasis [88]. It consists of a core particle, made up of alpha rings and beta rings, controlled by regulatory particles, which are made up of a number of proteins (Fig. 8a). The proteasome is an intricate molecular machine which requires complex regulation to unfold and deubiquitylate the substrate, and push it through the catalytic machinery located in the beta rings [89]. Necessarily, the proteasome undergoes large conformational changes during its operation. However, due to its size, such changes are very difficult to study. Recent work in the field of cryo-electron microscopy [90] has led to the discovery of intermediate conformers during the initial binding of ubiquitylated substrates. While the conformational changes in the regulatory particle are easily distinguishable (average backbone atom RMSD 10.4 $\AA$ ), the changes in the core particle are very subtle (average backbone atom RMSD $1.5 \AA$ ), due to the fact that all studied conformers refer to the initial phase of substrate binding.

Using ACC, we calculated atomic charges in these intermediate conformers of the $26 \mathrm{~S}$ proteasome [PDB: 4CR2, 4CR3, 4CR4]. The default ACC settings were used. The computation took 130s, and the complete results are available on the ACC web page at http://ncbr.muni.cz/ ACC/CaseStudy/Proteasome.

The calculation produced one set of atomic charges for each conformer of the $26 \mathrm{~S}$ proteasome. Since the proteasome is very large, we analyzed the residue charges, which are also reported by ACC, and subsequently the charges for the various subunits that make up the proteasome (details regarding the charge analysis on subunits can be found in the section Case study III of the Additional file 1). The first observation was that, during 


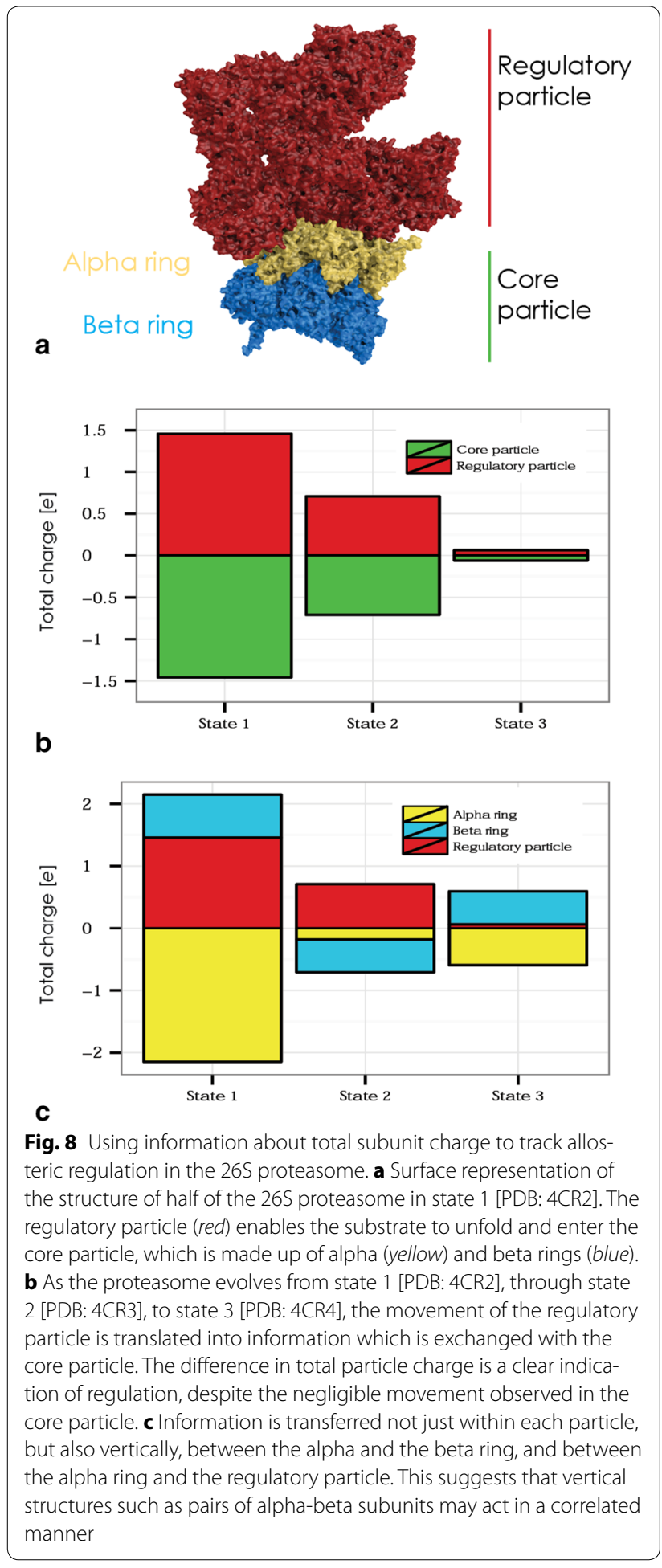

the conformational changes from state 1 , to state 2 , and then to state 3, a significant amount of electron density is transferred between the core particle and the regulatory particle (Fig. 8b). This suggests that, even though there is no significant movement observed for the core particle, allosteric information is exchanged with the core particle, and this information can be tracked at the electrostatic level. The next observation is that significant information is disseminated not only horizontally (within the alpha ring, or within the beta ring), but also vertically. In the overall transition it appears that the alpha ring loses electron density to the regulatory particle. By checking the intermediate state 2 it is possible to see that there is also transfer between the alpha ring and beta ring (Fig. 8c). This vertical shuttling of electron density within the core particle suggests that the activity of alpha and beta subunits may cross-correlate. Such phenomena have indeed been reported. For example, alpha5 and beta1 may translocate together [91], while knockdown of alpha1 leads to loss of chymotrypsin activity associated with beta5 [92]. Further analysis can even yield the residues involved in the allosteric regulation, as those residues which exhibit a high variation in total charge (e.g., approximately 10 sites on the Rpn-13 regulatory subunit).

It is important to note that the structures used in the EEM calculation were incomplete. Specifically, due to the size of these molecular machines, the resolution of the structures was too low to distinguish $\mathrm{H}$ atoms or even parts of residues. No modifications were made to the structures of the proteasome conformers prior to the EEM calculation. Thus, the charge distribution of each conformer is not expected to be physically relevant taken on its own. Moreover, the results are very likely affected by the polarizability exaggeration artifact of EEM, discussed in the Computational details section. Therefore, the analysis here focused on how the amount of charge in functional parts of the proteasome changes with the conformation. This case study shows how a brief calculation using only a crude structural approximation can give insight regarding allosteric regulation in large biomolecular complexes.

\section{Conclusions}

We present AtomicChargeCalculator (ACC), a webbased application for the calculation and analysis of atomic charges which respond to changes in molecular conformation and chemical environment. ACC also provides interactive facilities for statistical analysis and comparison of the results. We illustrate how direct analysis of atomic charges can give basic information about chemical reactivity in paracetamol, and how residue charges hold clues about biochemical relevance in the antimicrobial peptide protegrin-1. Additionally, ACC provides molecular structure files containing atomic charges, which can be used in further modelling studies. We illustrate how such data can be used for $\mathrm{p} K_{a}$ calculation using QSPR models. Another advantage of ACC is that it can 
handle any type of molecular system, regardless of size and chemical complexity, from drug-like molecules to biomacromolecular complexes with hundreds of thousands of atoms. We show how the direction and intensity of allosteric regulation can be tracked in large biomacromolecular systems like the proteasome even in the absence of high resolution structures. ACC thus caters to all fields of life sciences, from drug design to nano-carriers. AtomicChargeCalculator is freely available online at http://ncbr.muni.cz/ACC.

\section{Availability and requirements}

- Project name AtomicChargeCalculator

- Project home page http://ncbr.muni.cz/ACC

- Operating system(s) Web server - platform independent. Command line application-Windows, Linux, Mac OS

- Programming language C\#

- Other requirements For the web-server - modern internet browser with JavaScript enabled, WebGL support for 3D visualization. For the command line application - NET 4.0 for Windows based systems, Mono framework 3.10 or newer (http://www.monoproject.com) for other OS.

- License ACC license for the downloadable command line version.

- Any restrictions to use by non-academics Free of charge. No login requirement for running or accessing the results in the web server.

\section{Additional file}

Additional file 1. Full computational details, benchmark and limitations of ACC, along with supporting information for case studies I and III.

\section{Authors' contributions}

CMI, RSV and JK conceived the study, and participated in its design and coordination. DS designed and optimized the algorithms. DS, SG and LP implemented the web-application. CMI, DS, FLF, SG and PP performed extensive testing of the web-application. CMI and FLF prepared the documentation. CMI and TB performed the calculations for the case studies. CMI, TB and LP wrote the manuscript. All authors read and approved the manuscript.

\section{Author details}

${ }^{1}$ CEITEC-Central European Institute of Technology, Masaryk University, Kamenice 5, 62500 Brno, Czech Republic. ${ }^{2}$ National Centre for Biomolecular Research, Faculty of Science, Masaryk University, Kotlářská 2, 61137 Brno, Czech Republic. ${ }^{3}$ Faculty of Informatics, Masaryk University, Botanická 68a, 602 00 Brno, Czech Republic.

\section{Acknowledgements}

This work was supported by the Grant Agency of the Czech Republic [1325401S] and the European Community's Seventh Framework Programme [CZ.1.05/1.1.00/02.0068] from the European Regional Development Fund. Additional support was provided by the project "Employment of Newly Graduated Doctors of Science for Scientific Excellence" [CZ.1.07/2.3.00/30.0009] to CMI, co-financed from the European Social Fund and the state budget of the Czech Republic. The authors thank Mr. Ravi Ramos and Mr. Tomáš Raček for useful discussions.

\section{Competing interests}

The authors declare that they have no competing interests.

Received: 20 June 2015 Accepted: 8 October 2015

Published online: 22 October 2015

\section{References}

1. Giese B, Graber M, Cordes M (2008) Electron transfer in peptides and proteins. Curr Opin Chem Biol 12(6):755-759. doi:10.1016/j.cbpa.2008.08.026

2. Grodick MA, Muren NB, Barton JK (2015) DNA charge transport within the cell. Biochemistry 54(4):962-973. doi:10.1021/bi501520w

3. Li L, Wang L, Alexov E (2015) On the energy components governing molecular recognition in the framework of continuum approaches. Front Mol Biosci. doi:10.3389/fmolb.2015.00005

4. Zheng G, Xiao M, Lu XH (2005) QSAR study on the Ah receptor-binding affinities of polyhalogenated dibenzo-p-dioxins using net atomiccharge descriptors and a radial basis neural network. Anal Bioanal Chem 383:810-816. doi:10.1007/s00216-005-0085-7

5. Karelson M, Karelson G, Tämm T, Tulp I, Jänes J, Tämm K, Lomaka A, Deniss S, Dobchev D (2009) QSAR study of pharmacological permeabilities. Arkivoc 2009(2):218-238

6. Wood JS (1995) An X-ray determination of the electron distribution in crystals of hexapyridine-N-oxide cobalt(II) perchlorate and the electronic structure of the Co2+ ion. Inorganica Chimica Acta 229(1-2):407-415. doi:10.1016/0020-1693(94)04272-W

7. Belokoneva EL, Gubina YK, Forsyth JB, Brown PJ (2002) The chargedensity distribution, its multipole refinement and the antiferromagnetic structure of dioptase, $\mathrm{Cu}_{6}\left[\mathrm{Si}_{6} \mathrm{O}_{18}\right] \cdot 6 \mathrm{H}_{2} \mathrm{O}$. Phys Chem Min 29(6):430-438. doi:10.1007/s00269-002-0246-6

8. Schrödinger E (1926) An undulatory theory of the mechanics of atoms and molecules. Phys Rev 28(6):1049

9. Mulliken RS (1935) Electronic structures of molecules XI. Electroaffinity, molecular orbitals and dipole moments. J Chem Phys 3(9):573-585. doi:10.1063/1.1749731

10. Mulliken RS (1962) Criteria for the construction of good self-consistentfield molecular orbital wave functions, and the significance of LCAO-MO population analysis. J Chem Phys 36(12):3428. doi:10.1063/1.1732476

11. Löwdin P-O (1950) On the non-orthogonality problem connected with the use of atomic wave functions in the theory of molecules and crystals. J Chem Phys 18(3):365-375. doi:10.1063/1.1747632

12. Reed EA, Weinstock RB, Weinhold F (1985) Natural population analysis. J Chem Phys 83(2):735-746. doi:10.1063/1.449486

13. Bader RFW, Larouche A, Gatti C, Carroll MT, MacDougall PJ, Wiberg KB (1987) Properties of atoms in molecules: dipole moments and transferability of properties. J Chem Phys 87(2):1142-1152. doi:10.1063/1.453294

14. Hirshfeld FL (1977) Bonded-atom fragments for describing molecular charge densities. Theoretica Chimica Acta 44(2):129-138. doi:10.1007/BF00549096

15. Bultinck P, Van Alsenoy C, Ayers PW, Carbó-Dorca R (2007) Critical analysis and extension of the Hirshfeld atoms in molecules. J Cheml Phys. doi:10.1063/1.2715563

16. Breneman CM, Wiberg KB (1990) Determining atom-centered monopoles from molecular electrostatic potentials. The need for high sampling density in formamide conformational analysis. J Comput Chem 11(3):361373. doi:10.1002/jcc.540110311

17. Besler BH, Merz KM, Kollman PA (1990) Atomic charges derived from semiempirical methods. J Comput Chem 11:431-439. doi:10.1002/ jcc.540110404

18. Kelly CP, Cramer CJ, Truhlar DG (2005) Accurate partial atomic charges for high-energy molecules using class IV charge models with the MIDI! basis set. Theor Chem Acc 113(3):133-151. doi: 10.1007/s00214-004-0624-x

19. Manz TA, Sholl DS (2010) Chemically meaningful atomic charges that reproduce the electrostatic potential in periodic and nonperiodic materials. J Chem Theory Comput 6(8):2455-2468. doi:10.1021/ct100125x

20. Abraham RJ, Griffiths L, Loftus P (1982) Approaches to charge calculations in molecular mechanics. J Comput Chem 3(3):407-416. doi:10.1002/ jcc.540030316

21. Shulga DA, Oliferenko AA, Pisarev SA, Palyulin VA, Zefirov NS (2008) Parameterization of empirical schemes of partial atomic charge 
calculation for reproducing the molecular electrostatic potential. Doklady Chem 419(1):57-61. doi:10.1007/s10631-008-3004-6

22. Mortier WJ, Ghosh SK, Shankar S (1986) Electronegativity-equalization method for the calculation of atomic charges in molecules. J Am Chem Soc 108(15):4315-4320. doi:10.1021/ja00275a013

23. Rappé AK, Goddard WA III (1991) Charge equilibration for molecular dynamics simulations. J Phys Chem 95:3358-3363. doi:10.1021/ j100161a070

24. Nistor RA, Polihronov JG, Müser MH (2006) A generalization of the charge equilibration method for nonmetallic materials. J Chem Phys. doi:10.1063/1.2346671

25. Gasteiger J, Marsili M (1980) Iterative partial equalization of orbital electronegativity-a rapid access to atomic charges. doi:10.1016/0040-4020(80)80168-2

26. Cho K-H, Kang YK, No KT, Scheraga HA (2001) A fast method for calculating geometry-dependent net atomic charges for polypeptides. J Phys Chem A 105(17):3624-3634. doi:10.1021/jp0023213

27. Oliferenko AA, Pisarev SA, Palyulin VA, Zefirov NS (2006) Atomic charges via electronegativity equalization: generalizations and perspectives. doi:10.1016/S0065-3276(06)51004-4

28. Baekelandt B, Mortier W, Lievens J (1991) Probing the reactivity of different sites within a molecule or solid by direct computation of molecular sensitivities via an extension of the electronegativity equalization. J Am Chem Soc 113(18):6730-6734. doi:10.1021/ja00018a003

29. York DM, Yang W (1996) A chemical potential equalization method for molecular simulations. J Chem Phys 104(1):159. doi:10.1063/1.470886

30. Yang Z-Z, Wang C-S (1997) Atom-bond electronegativity equalization method. 1. Calculation of the charge distribution in large molecules. J Phys Chem A 101(35):6315-6321. doi:10.1021/jp9711048

31. Njo SL, Fan J, Van De Graaf B (1998) Extending and simplifying the electronegativity equalization method. J Mol Catal A Chem 134:79-88. doi:10.1016/S1381-1169(98)00024-7

32. Dias LG, Shimizu K, Farah JPS, Chaimovich H (2002) A simple method for the fast calculation of charge redistribution of solutes in an implicit solvent model. Chem Phys 282(2):237-243. doi:10.1016/ S0301-0104(02)00717-6

33. Chaves J, Barroso JM, Bultinck P, Carbó-Dorca R (2006) Toward an alternative hardness kernel matrix structure in the Electronegativity Equalization Method (EEM). J Chem Inform Model 46(4):1657-1665. doi:10.1021/ ci050505e

34. Ouyang Y, Ye F, Liang Y (2009) A modified electronegativity equalization method for fast and accurate calculation of atomic charges in large biological molecules. Phys Chem Chem Phys PCCP 11(29):6082-6089. doi:10.1039/b821696g

35. Verstraelen T, Van Speybroeck V, Waroquier M (2009) The electronegativity equalization method and the split charge equilibration applied to organic systems: Parametrization, validation, and comparison. J Chem Phys. doi:10.1063/1.3187034

36. Frisch M, Trucks G, Schlegel H, Scuseria G, Robb M, Cheeseman J, Scalmani G, Barone V, Mennucci B, Petersson G et al (2010) Gaussian 09 (revision a. 02), gaussian, inc., wallingford ct (USA). In: Naturforsch Z (ed) Vol. 10

37. Hutter J, lannuzzi M, Schiffmann F, Vandevondele J (2014) Cp2k: atomistic simulations of condensed matter systems. Wiley Interdiscip Rev Comput Mol Sci 4(1):15-25. doi:10.1002/wcms.1159

38. Manz TA, Sholl DS (2012) Improved atoms-in-molecule charge partitioning functional for simultaneously reproducing the electrostatic potential and chemical states in periodic and nonperiodic materials. J Chem Theory Comput 8(8):2844-2867

39. Verstraelen T, Vandenbrande S, Chan M, Zadeh FH, González C, Limacher PA, Horton AM (2013). http://theochem.github.com/horton/

40. Keith TA (2013) Aimall (version 13.05. 06). TK Gristmill Software, Overland Park

41. Marenich AV, Jerome SV, Cramer CJ, Truhlar DG (2012) Charge model 5: an extension of hirshfeld population analysis for the accurate description of molecular interactions in gaseous and condensed phases. J Chem Theory Comput 8(2):527-541. doi:10.1021/ct200866d

42. Malde AK, Zuo L, Breeze M, Stroet M, Poger D, Nair PC, Oostenbrink C, Mark AE (2011) An automated force field topology builder (ATB) and repository: Version 1.0. J Chem Theory Comput 7(12):4026-4037. doi:10.1021/ct200196m
43. Medeiros DDJ, Cortopassi WA, Costa França TC, Pimentel AS (2013) ITP adjuster 1.0: A new utility program to adjust charges in the topology files generated by the PRODRG server. J Chem. doi:10.1155/2013/803151

44. Wang J, Wang W, Kollman PA, Case DA (2006) Automatic atom type and bond type perception in molecular mechanical calculations. J Mol Graph Modelling 25(2):247-260. doi:10.1016/j.jmgm.2005.12.005

45. Vanquelef E, Simon S, Marquant G, Garcia E, Klimerak G, Delepine JC, Cieplak P, Dupradeau FY (2011) R.E.D. Server: a web service for deriving RESP and ESP charges and building force field libraries for new molecules and molecular fragments. Nucl Acids Res. doi:10.1093/nar/gkr288

46. Mukherjee G, Patra N, Barua P, Jayaram B (2011) A fast empirical GAFF compatible partial atomic charge assignment scheme for modeling interactions of small molecules with biomolecular targets. J Comput Chem 32(5):893-907. doi:10.1002/jcc.21671

47. Vainio MJ, Johnson MS (2007) Generating conformer ensembles using a multiobjective genetic algorithm. J Chem Inform Modeling 47(6):24622474. doi:10.1021/Ci6005646

48. Vařeková RS, Koča J (2006) Software news and update optimized and parallelized implementation of the electronegativity equalization method and the atom-bond electronegativity equalization method. J Comput Chem 27(3):396-405. doi:10.1002/jcc.20344

49. Dolinsky TJ, Nielsen JE, McCammon JA, Baker NA (2004) PDB2PQR: an automated pipeline for the setup of Poisson-Boltzmann electrostatics calculations. Nucl Acids Res. doi:10.1093/nar/gkh381

50. O'Boyle NM, Banck M, James CA, Morley C, Vandermeersch T, Hutchison GR (2011) Open babel: an open chemical toolbox. J Cheminform. doi:10.1186/1758-2946-3-33

51. Cho AE, Guallar V, Berne BJ, Friesner R (2005) Importance of accurate charges in molecular docking: Quantum Mechanical/Molecular Mechanical (QM/MM) approach. J Comput Chem 26(9):915-931. doi:10.1002/ jcc.20222

52. Anisimov VM (2010) Quantum-mechanical molecular dynamics of charge transfer. Kinetics Dynamics. doi:10.1007/978-90-481-3034-4 9

53. Nielsen JE, Gunner MR, García-Moreno EB (2011) The pK a Cooperative: a collaborative effort to advance structure-based calculations of $\mathrm{pK}$ a values and electrostatic effects in proteins. doi:10.1002/prot.23194

54. Baker CM (2015) Polarizable force fields for molecular dynamics simulations of biomolecules. Wiley Interdiscip Rev Comput Mol Sci 5(2):241254. doi:10.1002/wCms.1215

55. Heidler R, Janssens GOA, Mortier WJ, Schoonheydt RA (1996) Charge sensitivity analysis of intrinsic basicity of Faujasite-type zeolites using the electronegativity equalization method (EEM). J Phys Chem 100(50):19728-19734. doi:10.1021/jp9615619

56. Haldoupis E, Nair S, Sholl DS (2012) Finding MOFs for highly selective CO2/N2 adsorption using materials screening based on efficient assignment of atomic point charges. J Am Chem Soc 134(9):4313-4323. doi:10.1021/ja2108239

57. Bultinck P, Langenaeker W, Carbó-Dorca R, Tollenaere JP (2003) Fast calculation of quantum chemical molecular descriptors from the Electronegativity Equalization Method. J Chem Inform Comp Sci 43:422-428. doi:10.1021/ci0255883

58. Svobodová Vařeková R, Geidl S, lonescu C-M, Skřehota O, Bouchal T, Sehnal D, Abagyan R, Koča J (2013) Predicting p Ka values from EEM atomic charges. J Cheminform 5(1):18. doi:10.1186/1758-2946-5-18

59. Shimizu K, Chaimovich H, Farah JPS, Dias LG, Bostick DL (2004) Calculation of the dipole moment for polypeptides using the generalized born-electronegativity equalization method: results in vacuum and continuum-dielectric solvent. J Phys Chem B 108(13):4171-4177. doi:10.1021/jp037315w

60. Chen S, Yang Z (2010) Molecular dynamics simulations of a $\beta$-hairpin fragment of protein $\mathrm{G}$ by means of atom-bond electronegativity equalization method fused into molecular mechanics (ABEEM $\delta \pi / M M)$. Chin J Chem 28(11):2109-2118. doi:10.1002/cjoc.201090350

61. Ionescu CM, Svobodová Vařeková R, Prehn JHM, Huber HJ, Koča J (2012) Charge profile analysis reveals that activation of pro-apoptotic regulators bax and bak relies on charge transfer mediated allosteric regulation. PLoS Comput Biol. doi:10.1371/journal.pcbi.1002565

62. Chelli R, Procacci P, Righini R, Califano S (1999) Electrical response in chemical potential equalization schemes. J Chem Phys 111(18):8569. doi:10.1063/1.480198 
63. Warren Lee G, Davis JE, Patel S (2008) Origin and control of superlinear polarizability scaling in chemical potential equalization methods. J Chem Phy 128(14):144110. doi:10.1063/1.2872603

64. Verstraelen T, Pauwels E, De Proft F, Van Speybroeck V, Geerlings P, Waroquier M (2012) Assessment of atomic charge models for gas-phase computations on polypeptides. J Chem Theory Comput 8(2):661-676. doi:10.1021/ct200512e

65. van Duin ACT, Strachan A, Stewman S, Zhang Q, Xu X, Goddard WA (2003) ReaxFF SiO reactive force field for silicon and silicon oxide systems. J Phys Chem A 107(19):3803-3811. doi:10.1021/jp0276303

66. Puranen JS, Vainio MJ, Johnson MS (2009) Accurate conformationdependent molecular electrostatic potentials for high-throughput in silico drug discovery. J Comput Chem. doi:10.1002/jcc.21460

67. Bultinck P, Langenaeker W, Lahorte P, De Proft F, Geerlings P, Van Alsenoy C, Tollenaere JP (2002) The electronegativity equalization method II: applicability of different atomic charge schemes. J Phys Chem A 106(34):7895-7901. doi:10.1021/jp020547V

68. Bultinck P, Vanholme R, Popelier PLA, De Proft F, Geerlings P (2004) High-speed calculation of AIM charges through the electronegativity equalization method. J Phys Chem A 108(46):10359-10366. doi:10.1021/ jp0469281

69. Varekova RS, Jirouskova Z, Vanek J, Suchomel S, Koca J (2007) Electronegativity equalization method: parameterization and validation for large sets of organic, organohalogene and organometal molecule. Int J Mol Sci 8(7):572-582

70. Ionescu CM, Geidl S, Svobodová Vařeková R, Koča J (2013) Rapid calculation of accurate atomic charges for proteins via the electronegativity equalization method. J Chem Inform Model 53(10):2548-2558. doi:10.1021/ci400448n

71. Graham GG, Davies MJ, Day RO, Mohamudally A, Scott KF (2013) The modern pharmacology of paracetamol: therapeutic actions, mechanism of action, metabolism, toxicity and recent pharmacological findings. Inflammopharmacology 21(3):201-232. doi:10.1007/s10787-013-0172-x

72. Bertolini A, Ferrari A, Ottani A, Guerzoni S, Tacchi R, Leone S (2006) Paracetamol: new vistas of an old drug. CNS Drug Rev. 12(3-4):250-275. doi:10.1111/j.1527-3458.2006.00250.x

73. Svobodová Vařeková R, Geidl S, lonescu CM, Skřehota O, Kudera M, Sehnal D, Bouchal T, Abagyan R, Huber HJ, Koča J (2011) Predicting pKa values of substituted phenols from atomic charges: comparison of different quantum mechanical methods and charge distribution schemes. $J$ Chem Inform Modeling 51(8):1795-1806. doi:10.1021/ci200133w

74. Ugur I, Marion A, Parant S, Jensen JH, Monard G (2014) Rationalization of the pKa values of alcohols and thiols using atomic charge descriptors and its application to the prediction of aminoacid pKa's. J Chem Inform Modeling. doi:10.1021/ci500079w

75. Dastmalchi S, Rashidi M, Rassi M (1995) Simultaneous determination of the pka and octanol/water partition coefficient (pm) of acetaminophen. J Sch Pharm Med Sci Univ Tehran 4:7-14

76. $\mathrm{NCl}$ Open Database Compounds. National Cancer Institute. http://cactus. nci.nih.gov/. Accessed Aug 2015

77. Howard P, Meylan W (1999) Physical/chemical property database (PHYSPROP). Syracuse Research Corporation, Environmental Science Center, North Syracuse

78. Geidl S, Svobodová Vařeková R, Bendová V, Petrusek L, lonescu CM, Jurka Z, Abagyan R, Koča J (2015) How does the methodology of 3D structure preparation influence the quality of $\mathrm{p} \mathrm{K}$ a prediction? J Chem Inform Modeling 55(6):1088-1097. doi:10.1021/ci500758w

79. Bellm L, Lehrer RI, Ganz T (2000) Protegrins: new antibiotics of mammalian origin. Exp Opin Investig Drugs 9(8):1731-1742 doi:10.1517/13543784.9.8.173
80. Steinberg DA, Hurst MA, Fujii CA, Kung AHC, Ho JF, Cheng FC, Loury DJ, Fiddes JC (1997) Protegrin-1: a broad-spectrum, rapidly microbicidal peptide with in vivo activity. Antimicrob Agents Chemotherap 41(8):1738-1742

81. Dong N, Zhu X, Chou S, Shan A, Li W, Jiang J (2014) Antimicrobial potency and selectivity of simplified symmetric-end peptides. Biomaterials 35(27):8028-8039. doi:10.1016/j.biomaterials.2014.06.005

82. Mohanram H, Bhattacharjya S (2014) Cysteine deleted protegrin-1 (CDP1): anti-bacterial activity, outer-membrane disruption and selectivity. Biochimica et Biophysica Acta (BBA) General Subjects 1840(10):3006-3016. doi:10.1016/j.bbagen.2014.06.018

83. Ostberg N, Kaznessis Y (2005) Protegrin structure-activity relationships: using homology models of synthetic sequences to determine structural characteristics important for activity. Peptides 26(2):197-206. doi:10.1016/j.peptides.2004.09.020

84. Fahrner RL, Dieckmann T, Harwig SSL, Lehrer RI, Eisenberg D, Feigon J (1996) Solution structure of protegrin-1, a broad-spectrum antimicrobial peptide from porcine leukocytes. Chem Biol 3(7):543-550. doi:10.1016/ S1074-5521(96)90145-3

85. Bolintineanu DS, Langham AA, Davis HT, Kaznessis YN (2007) Molecular dynamics simulations of three protegrin-type antimicrobial peptides: interplay between charges at the termini, $\beta$-sheet structure and amphiphilic interactions. doi:10.1080/08927020701393481

86. Langham AA, Khandelia H, Schuster B, Waring AJ, Lehrer RI, Kaznessis YN (2008) Correlation between simulated physicochemical properties and hemolycity of protegrin-like antimicrobial peptides: Predicting experimental toxicity. Peptides 29(7):1085-1093. doi:10.1016/j. peptides.2008.03.018

87. Lai JR, Huck BR, Weisblum B, Gellman SH (2002) Design of non-cysteinecontaining antimicrobial $\beta$-hairpins: Structure-activity relationship studies with linear protegrin-1 analogues. Biochemistry 41(42):12835-12842. doi:10.1021/bi026127d

88. Bedford L, Paine S, Sheppard PW, Mayer RJ, Roelofs J (2010) Assembly, structure, and function of the $26 \mathrm{~S}$ proteasome. doi:10.1016/j. tcb.2010.03.007

89. Gallastegui N, Groll M (2010) The 265 proteasome: assembly and function of a destructive machine. doi:10.1016/j.tibs.2010.05.005

90. Unverdorben P, Beck F, Śledź P, Schweitzer A, Pfeifer G, Plitzko JM, Baumeister W, Förster F (2014) Deep classification of a large cryo-EM dataset defines the conformational landscape of the $26 \mathrm{~S}$ proteasome. Proc Natl Acad Sci USA 111(15):5544-5549. doi:10.1073/pnas.1403409111

91. O'Hara A, Howarth A, Varro A, Dimaline R (2013) The role of proteasome beta subunits in gastrin-mediated transcription of plasminogen activator inhibitor-2 and regenerating protein1. PLoS One. doi:10.1371/journal. pone.0059913

92. Cron KR, Zhu K, Kushwaha DS, Hsieh G, Merzon D, Rameseder J, Chen CC, D'Andrea AD, Kozono D (2013) Proteasome inhibitors block DNA repair and radiosensitize non-small cell lung cancer. PLoS One. doi:10.1371/ journal.pone.0073710

\section{Publish with ChemistryCentral and every scientist can read your work free of charge \\ "Open access provides opportunities to our colleagues in other parts of the globe, by allowing anyone to view the content free of charge."}

W. Jeffery Hurst, The Hershey Company.

- available free of charge to the entire scientific community

- peer reviewed and published immediately upon acceptance

- cited in PubMed and archived on PubMed Central

- yours - you keep the copyright

Submit your manuscript here:

http://www.chemistrycentral.com/manuscript/ 